\title{
Autonomous Optimization of UWB Link Access
}

\author{
María. D. Pérez-Guirao, Ralf Luebben and Thomas Kaiser
}

Abstract - We present a novel approach for interference management in low data rate IR-UWB networks that enables concurrent transmissions at full power while allows each source to independently adapt its pulse rate (transmitted pulses per second) to mitigate multi-user interference. The work is motivated by the fact that the distributed adaptation of IR-UWB pulse rate has not been sufficiently addressed in the literature before. Existing approaches rely on the presence of a central authority or assume a definite receiver technique. Our approach enables users to share the communication medium in an efficient way compatible with individual QoS requirements and it is independent of any particular modulation scheme or receiver technique.

Index Terms - IR-UWB, MUI, interference management, link adaptation

\section{Introduction}

The growing request for low to medium data rate low cost networks raises the interest in wireless sensor technology. For instance, in the field of industrial and logistic applications, in order to improve the processes' efficiency the tight monitoring of goods, tools, and machinery (as to their state and position) is required. For such applications to be available, reliable and low power communication and, on the other hand, robust but accurate localization capabilities are required. Ultra-Wideband (UWB) radio, particularly with impulse radio transmission (IR-UWB), is a key technology for the development of low power, low data rate (LDR), indoor/outdoor joint communication and localization applications.

In order to materialize the benefits of IR-UWB in wireless sensor networks (WSN), the design of the medium access control (MAC) layer plays a significant role. Particularly challenging is the management of interference either from the outside world or from within the WSN (multi-user interference). For low cost WSN complex and expensive interference mitigation techniques such as multi-user detection are unfeasible. Advantegeously, its inherent high temporal resolution furnishes IR-UWB with natural robustness against multi-user interference (MUI) and multipath fading. Under low to moderate $\left(<0.01\right.$ nodes $\left./ \mathrm{m}^{2}\right)$ node density, this inherent robustness makes uncoordinated access to the shared medium possible and interference management mechanisms unnecessary. However, the ever increasing demand for real-world sensory data leads to high density WSN $\left(>0.01\right.$ nodes $\left./ \mathrm{m}^{2}\right)$ and makes MUI management indispensable.

In this work we contribute with the design, implementation and investigation of a novel interference management strategy to compensate MUI in highly loaded low power LDR IRUWB networks.

\section{System model}

We consider a low power LDR sensor positioning and identification network (SPIN) like those found in production/logistics business applications. The network comprises up to a hundred of heterogeneous nodes (e.g. sensors and tags) attached to mobile devices (such as material flowing through the factory floor, transport and monitoring units, etc), which transmit data packets via an IR-UWB communication link to one common receiver, hereafter referred to as the cluster head $(\mathrm{CH})$. The traffic in the network is expected to be application traffic, for instance identification and sensor data, with relaxed delay constraints. The packet inter-arrival process at each sensor node is assumed to be exponential distributed and mutually independent. We consider a maximum information data rate per sensor node of $50 \mathrm{kbits} / \mathrm{s}$.

The network is modeled as a set of $|L|$ distinct node pairs (links), $L=\{1,2, \ldots,|L|\}$. Data is transmitted using sequences of pulses with an average inter-pulse distance $\left(T_{f}\right)$ much larger than the pulse duration $\left(T_{p}\right)$, such that a low duty cycle signal is obtained and uncoordinated channel access is allowed with pseudo-randomly selected time hopping (TH) codes, that determine the temporal position of the transmitted pulses. The use of TH codes further increases the inherent resilience of the IR-UWB technology to MUI [1].

We consider a system bandwidth of $\mathrm{B}=1.5 \mathrm{GHz}$ and $\mathrm{TH}-$ UWB signals with average frame length, $T_{f}$, in the range from 1 to $100 \mu \mathrm{s}$. This corresponds to low to moderate pulse repetition frequencies in the range of $10 \mathrm{kHz}$ to $1 \mathrm{MHz}$. The pulse repetition frequency is defined as the inverse of the average frame length $\mathrm{f}_{r}=1 / \mathrm{T}_{f}$.

The energy per transmitted pulse, $E_{p}^{t x}$, is the same for all transmitters in the system. It is chosen such that the transmitter with the highest pulse repetition frequency, i.e. $f_{r}=1$ $\mathrm{MHz}$, completely exploits the power spectral density limit of $-41.3 \mathrm{dBm} / \mathrm{MHz}$ imposed by the FCC. Additionally, it is assumed that each source node can autonomously adapt its average pulse repetition frequency $\left(f_{r}\right)$. Note that controlling the source's $f_{r}$ is equivalent to controlling it's channel access rate in terms of pulses per second (Pps); with fixed $E_{p}^{t x}$, this is also equivalent to controlling the source's average transmitted power.

For analytical convenience, we assume an additive white Gaussian noise (AWGN) channel and that radio propagation follows a log-normal distance path loss model without shadowing variations. At the air interface, the superframe structure described in Section 5.4.1 of the IEEE 802.15.4a standard is assumed. The $\mathrm{CH}$ periodically broadcasts beacon frames (BFs) containing timing information to support node synchronisation; the BFs can also be used to identify the network and to provide the sensor nodes with network configuration information. Two consecutive BFs delimite a contention access period (CAP). During the CAP, sensor nodes that want to transmit to the $\mathrm{CH}$ do so following the ALOHA scheme, that is without previously sensing the medium or waiting for a specific time slot. 


\section{Distributed compensation of MUI in IR-UWB Networks}

Interference compensation is a challenging task involving several aspects across the physical, data link and network layers of the communication systems. In the sequel we focus on interference management at the data link layer.

In a similar way as with CDMA, IR-UWB systems suffer from the well-known near-far effect. In practice, the number of harmful near interferers in a dense sensor network, as expected in industrial settings, can be high and lead to high local cumulative traffic loads (measured in Pps). In the recent past, research efforts have been dedicated to evaluate and quantify the limitations of IR-UWB when considering high traffic loads [2] and even applications requesting quality of service (QoS) [3]. In both situations, the resilience against MUI provided by the physical layer was shown to be insatisfactory and the introduction of additional interference management mechanisms was recommended.

In CDMA systems, a well-known interference management technique is to control the nodes' transmit power [4] However, for wireless networks in the linear regime, as IRUWB networks, a broadly accepted result claims that the only necessary power control is to let every node either transmit with full power or not transmit at all [5]. According to [5], the MAC layer should concentrate on interference management techniques such as scheduling and rate adaptation. Particularly, for low power networks the optimal MAC layer design should follow an "all at once" scheduling and adapt the transmission rates to interference. In IR-UWB networks, rate control can be achieved by adapting the channel coding rate, the modulation order or the processing gain. Our work focuses on rate adaptation by means of distributed processing gain adaptation. As far as we know, with the exception of the work in [6], which requires a particular receiver technique, this topic has not been addressed in the literature before. The processing gain of IR-UWB is twofold: the number of pulses per symbol $\left(N_{s}\right)$ and the average frame length $\left(T_{f}\right)$. The first theoretical considerations about the performance tradeoff between the two types of IR-UWB processing gain were published by Fishler and Poor in [7]. In terms of robustness against MUI and assuming that the energy per transmitted pulse is constant for all users, the authors showed that a reduction of the signal's duty cycle by extending the average frame length is preferable than an increase in the number of pulses transmitted per symbol.

Based on that result, we developed a channel access mechanism that incite transmitters to regulate their impulsive emissions by decreasing their pulse repetition frequency $\left(f_{r}\right)$ in order to avoid high MUI situations. This channel access mechanism is referred to as Pulse Rate Control (PRC) and can be realised in form of a rate adaptation function whose goal is the improvement of the link/network throughput while satisfying certain per link QoS requirement. PRC is independent of a particular modulation scheme or receiver technique. Furthermore, it can be combined with conventional adaptive channel coding.

\section{Pulse rate control}

This section deals with the modeling and analytical evaluation of PRC. Initially, PRC is formulated as a resource allocation problem seeking to coordinate the pulse repetition frequency of the individual links in order to optimize the overall network performance, in terms of a certain objective function, while statisfying a set of per-link QoS constraints. Afterwards, an in order to solve the problem in a distributed manner, two different approaches are presented. The first approach exploits the subjacent strategic interaction of the problem to reformulate it as a non-cooperative game with pricing. The second approach consists in a simple heuristic algorithm motivated by the request of inexpensive devices with low power consumption.

\subsection{Problem formulation}

The goal of the PRC approach is to determine the vector of pulse repetition frequencies, $f_{r}=\left(f_{r 1}, f_{r 2}, \ldots, f_{r|L|}\right)$ that solves the following constrained maximization problem:

$$
\begin{aligned}
& \max _{f_{r}} \sum_{i=1}^{|L|} \log \left(r_{i}\left(f_{r}\right)\right) \\
& \text { subject to: (a) } P_{e i} \leq \beta_{i} \forall i \in L \text { and (b) } \\
& f_{r i} \in F_{r i}=\left[f_{r i}^{\min }, f_{r i}^{\max }\right], \forall i \in L
\end{aligned}
$$

The objective function corresponds to the sum over all links of the logarithm of the links' data rate $\left(r_{i}\right)$ and embodies the goal of trading aggregate network throughput off for fairness. The constraints reflect the fact that the per-link average bit error rate (BER), $P_{e i}$, is bounded by certain application specific threshold $\beta_{i}$, and that the set of actions available to each sensor node, $F_{r i}$, is bounded. The net data rate per link is given in equation (2)

$r_{i}\left(f_{r}\right)=r_{i}^{r a w}\left(1-P_{e i}\left(f_{r-i}\right)\right)[\mathrm{bit} / \mathrm{s}]$

Where $f_{r}=\left(f_{r 1}, \ldots, f_{r i-1}, f_{r i+1} \ldots, f_{r|L|}\right)$ so that $f_{r}=\left(f_{r i}, f_{r-i}\right)$. The raw data rate per link, $r_{i}^{\text {raw }}$, can be controlled by adapting the channel coding rate $\left(R_{i}\right)$, the modulation order $\left(m_{i}\right)$ or the processing gain, that is the number of pulses per symbol $\left(N_{s}^{i}\right)$ and/or the average pulse repetition frequency $\left(f_{r i}\right)$. Equation (3) depicts the dependency of the raw data rate on these parameters. For the sake of simplicity and without loss of generality we consider $N_{s}^{i}=1, \forall i \in L$.

$r_{i}^{r a w}=\frac{f_{r i}}{N_{s}^{i}} R_{i} \log _{2}\left(m_{i}\right)[\mathrm{bit} / \mathrm{s}]$

PRC is a nonlinear optimization problem, since the objective function and the constraints on the BER are nonlinear. The classical optimization theory has no general method for solving a general nonlinear optimization problem, but several different approximation techniques (reformulation linearization, integer approximation, etc). The majority involves algorithms with a central point of computation. In our work we seek for autonomous algorithms capable of solving the problem in a distributed way by the sensor nodes. To this end, game theory (GT) represents an alternative to classical optimization theory and it is used in the next section to solve problem in a distributed manner.

\subsection{PRC game}

While adaptive modulation and coding are local decisions to a sender-receiver pair, PRC involves a strategic interaction among different neighbouring links. The average probability of pulse collision at the $i$-th receiver, and thus its average BER, does not depend on its own link's pulse rate, $f_{r i}$, but on the pulse rates of concurrent transmitters in its vicinity, $f_{r-i}[8]$.

We can exploit this strategic interaction and reformulate (1) into a non-cooperative game with pricing. Now each link is modeled as a selfish player with the aim of maximizing its own objective function

$v_{i}\left(f_{r}\right)=\log \left(r_{i}\left(f_{r}\right)\right)-\pi_{i}\left(f_{r}\right) f_{r i}$ 
The logarithmic term reflects the level of the player's satisfaction from consuming the resource Pps (directly related to an increase of its net data rate), while the right term in equation (4) is a linear function of the link's pulse rate, that reflects the price that player has to pay due to the interference (pulse collisions) it generates.

In the game, the $\mathrm{CH}$ calculates and broadcasts in each beacon frame a common cost factor for all players, i.e. $\pi_{i}=\pi_{C H} \forall i \in L$. Therewith, the $\mathrm{CH}$ can discourage the overuse of the resource Pps and keep the level of interference compatible with the per-link QoS requirements. In the scenario considered in this article, the PRC game can be proved to be an ordinal potential game and has therefore at least one Nash Equilibrium (NE) [9]. In a potential game although players act selfishly, they actually implicitly cooperate towards a common system goal represented by the potential function. The potential function of the PRC game is given in (5) It combines the original objective function of problem (1) with a linear price term that aims at selecting the NE that satisfies the original problem's constraints. A detailed analysis of the game's equilibrium, the computation of the price factor in two different network topologies, namely centralized and peer-to-peer, and a practical distributed algorithm that reproduces the player's behavior in the game can be found in [10].

$\Phi\left(f_{r}\right)=\sum_{i \in L} \log \left(r_{i}\left(f_{r}\right)\right)-\pi_{C H} \sum_{i \in L} f_{r i}$

\subsection{Towards minimum complexity Algorithms}

The main drawback of the previous approach is the fact that in order to find its optimal (best response) $f_{r i}$ each player has to compute its objective function over the whole action space $F_{r i}$ Particularly, when thinking about embedded hardware implementations linear arithmetic functions are prefered to nonlinear $\log (\cdot)$ functions. Also, energy efficiency is a driver towards the design of lowest complexity algorithms for WSN

This section proposes an algorithm inspired by the randomly, pairwise, learning/selection process of evolutionary algorithms (EA) [11]. It is intended as a low complexity alternative to the PRC game formulation.

The algorithm is called E-PRC (Evolutionary PRC) and consists of the following steps:

1. Initialization: Each link $i \in L$ chooses an initial pulse repetition frequency $f_{r i}(0) \in F_{r i}$

2. Pairwise Pulse Rate Revision: At each iteration $k$, link $i$ randomly selects a neighbouring link $j$ and updates its $f_{r i}$ according to the rule in, where $\Delta f_{r}$ is a constant equal to $1 \mathrm{kHz}$.

$$
f_{r i}(k+1)=\left\{\begin{array}{l}
f_{r i}(k)+\Delta f_{r}, \text { if } P_{e j} \leq \beta_{j} \\
f_{r i}(k)-\Delta f_{r}, \text { if } P_{e j}>\beta_{j}
\end{array}\right.
$$

\section{Performance Evaluation}

We present here simulations results obtained with the packetbased network simulator OMNeT $++[12]$. Our simulation model supports M-ary pulse position modulation (PPM) schemes with pseudorandom TH codes and computes MUI in a probabilistic manner. For each data packet a packet error rate (PER) is computed as a function of the received power, interference from concurrent transmissions, and thermal noise. Data packets have a fixed length of 400 bits. The upper bound for the per-link PER is $18 \%$, which corresponds

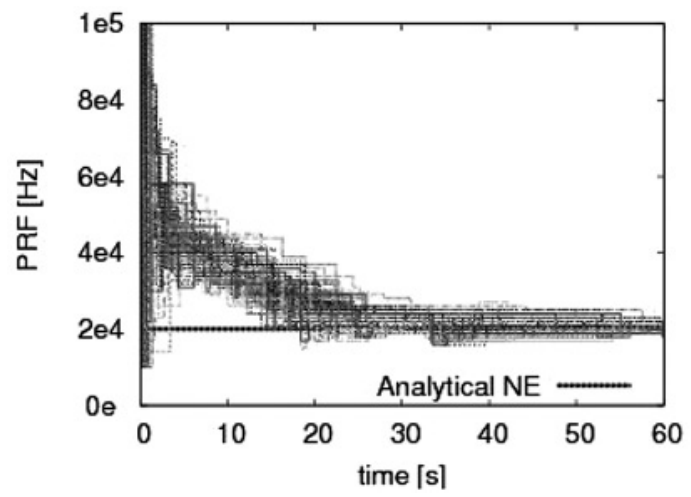

Fig. 1: Convergence to the game's NE when the information data rate per sensor node is $15 \mathrm{kbits} / \mathrm{s}$.

The simulated scenario accounts for a square simulation field with dimensions $30 \mathrm{~m}$ x $30 \mathrm{~m}$. In this field, $100 \mathrm{UWB}$ sensor nodes are considered: 5 are collocated at fixed positions, 75 move along a production line at $5 \mathrm{~m} / \mathrm{s}$ while the rest randomly moves at the same speed within the simulation field. In order to test the interference compensation functionality of the PRC strategy, its performance under increasing offered system load is compared to the one obtained with an ALOHA protocol without feedback channel and fixed $f_{r i}=1$ $\mathrm{MHz} \forall i \in L$. IEEE 802.15.4a proposes ALOHA with optional feedback channel as the standard MAC protocol for LDR IR-UWB sensor networks. We renounce to use a feedback channel in order to keep the power consumption at the sensor nodes low.

Simulation results have confirmed the existence of a unique NE for the PRC game and that the distributed algorithm in [10] converges to it under both traditional best-response and stochastic better-response update rules. Fig. 1 illustrates the convergence to the game's NE with better-response dynamics and an information data rate per sensor node set to $15 \mathrm{kbit} /$ s. With that per-link information data rate, the offered system load would not be compliant with the problem's QoS requirements. Thus, $\mathrm{PRC}$ reduces the links' channel access rates to a sustainable level (approx. $20 \mathrm{kHz}$ per link), which is identified by the common cost factor $\pi_{C H}$.

Fig. 2 depicts a performance comparison between ALOHA with and without PRC in a scenario with increasing offered load. The performance is reflected in terms of aggregate network throughput and average PER, both measured at the $\mathrm{CH}$. The PRC approach is able to limit the system load to a level compatible with the QoS requirements. Notice that the regulative effect of the PRC approach limits the maximum possible aggregate network throughput to approximately 1.25 Mbits/s, while ALOHA with fixed $f_{r}$ cannot guarantee the design QoS constraint and breaks down as the information data rate per link increases to $30 \mathrm{kbit} / \mathrm{s}$.

Fig. 3 contrasts the performance of the game theoretical (APRC) and the heuristic (E-PRC) approaches, again in terms of aggregate network throughput and average PER, under increasing offered load. Except for larger data rates (30 $\mathrm{kbit} / \mathrm{s}$ and $50 \mathrm{kbit} / \mathrm{s}$ ) where A-PRC achieves a slightly higher cumulative throughput at the cost of a higher PER, both approaches reach the same average performance. Thus, EPRC is an appropriate low complexity alternative to the classical game theoretical formulation of the PRC concept. Its main drawback is its slow convergence speed, which is inherent to the class of EA. While in the classical game theoretical approach each link is able to predict its optimal $f_{r}$ level and to directly choose it (best-response update), the EPRC algorithm simply reacts to environmental changes with small, bounded, fluctuations. However, in a realistic environment, where observations may be corrupted by noise, the slow 

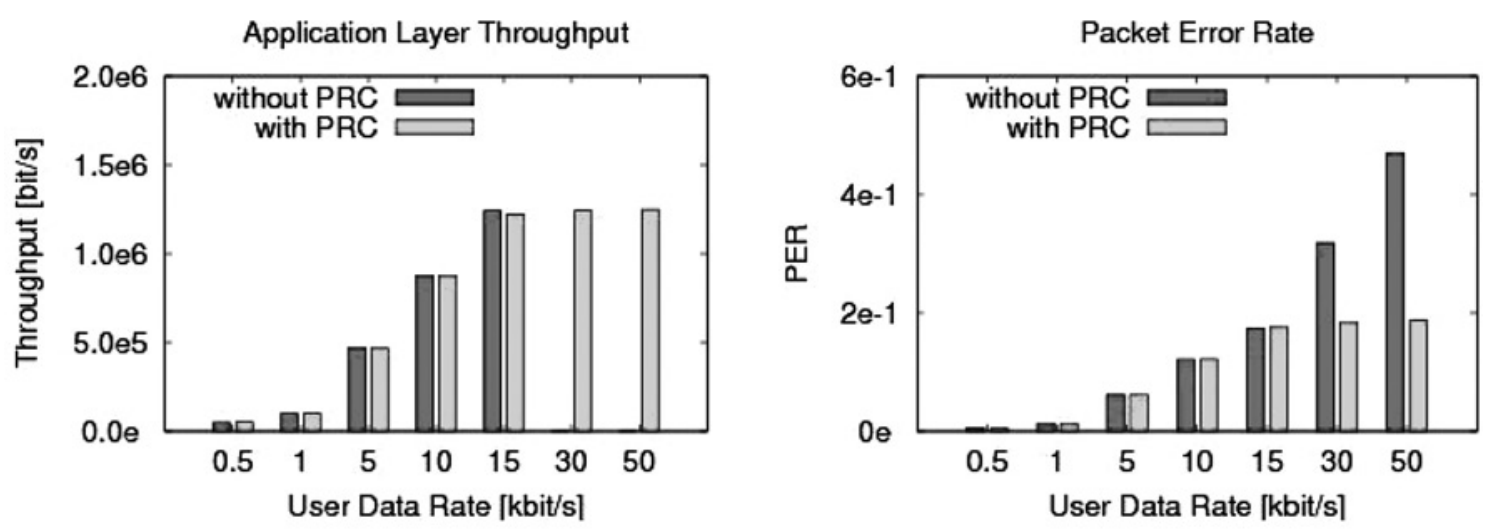

Fig. 2: Performance comparison of ALOHA with and without PRC.
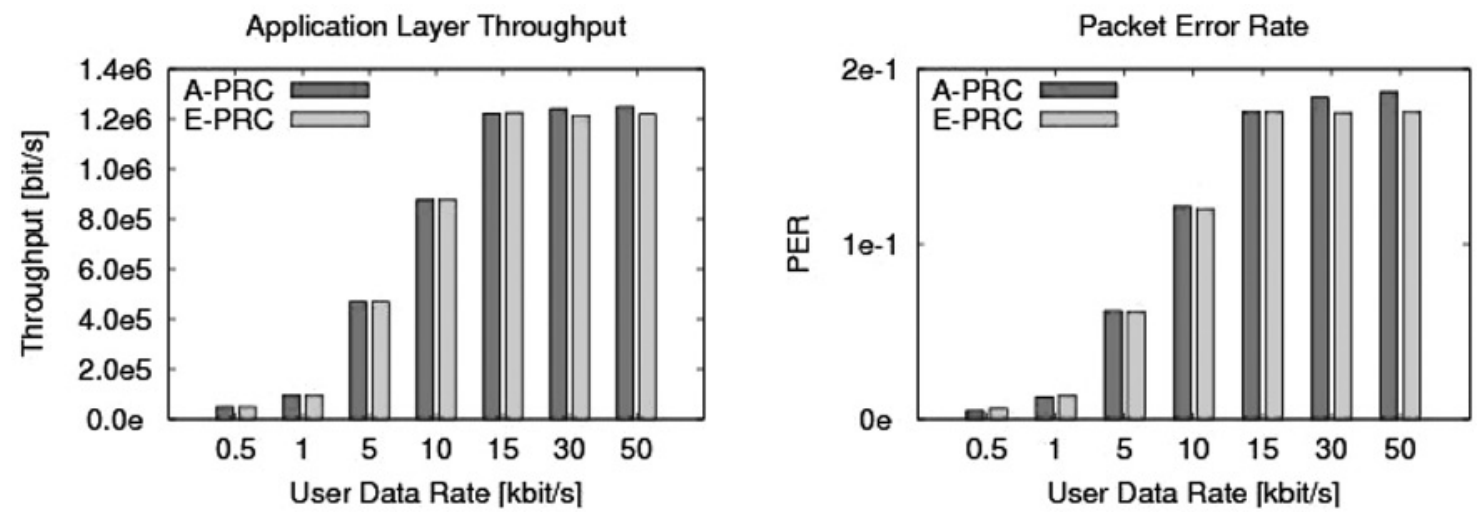

Fig. 3: Performance comparison of A-PRC vs. E-PRC.

adaptation process of E-PRC has the positive effect of making the system more robust against "bad observations".

\section{Conclusion}

In this article we discuss a novel strategy for impulsive interference management for LDR low power IR-UWB networks that exploits pulse rate (transmitted pulses per second) adaptation. The strategy enables concurrent transmissions at full power while allows each source to independently adapt its pulse rate. It is independent of a particular modulation scheme or receiver technique and could be combined with conventional adaptive channel coding. We have presented two distributed approaches for its implementation and investigated their performance via simulation. Our results show that PRC is an appropriate means for interference mitigation and network throughput optimization with QoS constraints in IR-UWB networks which are under high load conditions and have preferably low mobility.

\section{References}

[1] Win, M.Z. and Scholtz, R.A. "Ultra-Wide Bandwidth TimeHopping Spread-Spectrum Impulse Radio for Wireless MultipleAccess Communications". IEEE Transactions on Communications, vol. 48(4), pp. 679-689, Apr 2000.

[2] de Nardis, L. , Giancola, G. and di Benedetto, M.-G., "Performance Analysis of Uncoordinated Medium Access Control in Low Data Rate UWB Networks", 2nd International Conference on Broadband Networks, 2005.

[3] August, N.J., Chung, W. and Ha, D. S., "Distributed MAC Protocols for UWB Ad Hoc and Sensor Networks", IEEE Radio and Wireless Symposium, 2006.

[4] Muqattash, A. and Krunz, M., "CDMA-based MAC Protocol for Wireless Ad Hoc Networks". Proc. 2003 4th ACM international symposium on Mobile ad hoc networking \& computing, 2003, pp. $153-164$.

[5] Radunovic B. and J.-Y. Le Boudec, "Optimal power control, scheduling, and routing in UWB networks", IEEE Journal on Selected Areas in Communications, Vol. 22, No. 7. (2004), pp. 1252 1270.

[6] Le Boudec, J.-Y. , Merz, R. , Radunovic, B. and Widmer, J. , ’DCCMAC: a Decentralized MAC Protocol for 802.15.4a-like UWB Mobile Ad-Hoc Networks based on Dynamic Channel Coding”. In Proc. First International Conference on Broadband Networks, BroadNets, 2004 pp. 396-405.

[7] Fishler, E. and Poor, V., "On the Tradeoff between two Types of Processing Gains", IEEE Transactions on Communications, Vol. 53 (2005), pp. 1744-1753.

[8] Weisenhorn, M. and Hirt, W., "Uncoordinated Rate-Division Multiple-Access Scheme for Pulsed UWB Signals", IEEE Transactions on Vehicular Technology, Vol. 54 (5), pp. 1646-1662, Sept. 2005.

[9] Monderer, D. and Shapley, S., "Potential Games", Games and Economics Behaviour, Vol. 14, pp.:124-143, 1996.

[10] Pérez-Guirao, M.-D., Luebben. R, Kaiser, T., "Cross-Layer, Cognitive, Cooperative Pulse Rate Control for Autonomous, Low-Power, IR-UWB Networks", Dissertation, Leibniz Universität Hannover, 2008.

[11] Hofbauer, J. and Sigmund, K., "Evolutionary Game Dynamics". Bulletin of theAmerican Mathematical Society, Vol. 40(4), pp.479$519,2003$.

[12] http://www.omnetpp.org/, Omnet++, discrete event simulation system.

Authors:

M. Dolores Pérez-Guirao

Institut für Kommunikationstechnik

Leibniz Universität Hannover

E-mail:Dolores.perez@ikt.uni-hannover.de

Ralf Luebben, Thomas Kaiser

Intitut für Kommunikationstechnik

Leibniz Universität Hannover

This work was supported by DFG Priority Program UKoLoS SPP1202 